\title{
Preparation of a toothpaste containing theobromine and fluoridated bioactive glass and its effect on surface micro-hardness and roughness of enamel
}

\author{
Imran FAROOQ ${ }^{1}$, Abdul Samad KHAN², Imran Alam MOHEET ${ }^{3}$ and Emad ALSHWAIMI ${ }^{2}$ \\ ${ }^{1}$ Department of Biomedical Dental Sciences, College of Dentistry, Imam Abdulrahman Bin Faisal University, Dammam, Saudi Arabia \\ ${ }^{2}$ Department of Restorative Dental Sciences, College of Dentistry, Imam Abdulrahman Bin Faisal University, Dammam, Saudi Arabia \\ ${ }^{3}$ Azra Naheed Dental College, Superior University, Lahore, Pakistan \\ Corresponding author, Imran FAROOQ; E-mail: drimranfarooq@gmail.com
}

\begin{abstract}
The aim was to synthesize a toothpaste and analyze its effect on surface micro-hardness and roughness of enamel. Basic paste was prepared by using basic ingredients. Theobromine $(0.2 \mathrm{wt} \%)$ and laboratory synthesized fluoridated-bioactive glass (F-BG, $4 \mathrm{wt} \%)$ were added to it. Post-demineralization, 36 enamel blocks were divided into six groups that were brushed with their respective toothpaste+artificial saliva (AS): group 1 (control): basic paste; group 2: basic paste+theobromine; group 3: commercial theobromine toothpaste; group 4: commercial BG toothpaste; group 5: basic paste+F-BG; and group 6: basic paste+theobromine+F-BG. On micro-hardness analysis, group 6 performed best, followed by group 4. Surface roughness results showed the maximum decrease in roughness values for group 6 , followed by group 5 . Treatment with toothpaste composition containing theobromine $+\mathrm{F}-\mathrm{BG}$ resulted in the enamel's increased micro-hardness and decreased surface roughness.
\end{abstract}

Keywords: Biomaterial, Dental enamel, Fluoride, Theobromine, Toothpastes

\section{INTRODUCTION}

The oral cavity routinely goes through cycles of demineralization and remineralization ${ }^{1)}$. White spot lesions on the enamel surface caused by loss of minerals during the carious process are reversible ${ }^{2}$. The recommended method to prevent and reverse initial lesions is to use toothpastes, mouthwashes, and gels with appropriate active ingredients ${ }^{3)}$.

Toothpastes are everyday oral products that contain an active ingredient along with other basic components ${ }^{4}$. Various active ingredients that have a role in promoting enamel remineralization are added to toothpastes, the most common among these being fluoride and bioactive glasses $(\mathrm{BG})^{4}$. The role of fluoride in reducing dental caries is well documented in the literature ${ }^{5}$. Fluoride ions help to reduce crystal volume by replacing hydroxyl in the apatite structure, resulting in overall stability and structural strength ${ }^{6}$. This replacement changes the hydroxyapatite (HA) into fluorapatite (FA), which is more resistant to dental caries and acid attacks ${ }^{7}$. BG was first introduced by Larry Hench in 1969 for bone osseointegration, and it contains calcium, sodium, and phosphosilicate ${ }^{8)}$. The tremendous bone-bonding ability of BG could be attributed to HA layer formation on bone's surface when BG dissolves in an aqueous solution ${ }^{9}$.

The BG is now routinely used in oral products like toothpastes, as it has similar composition to dental enamel $^{4}$. The traditional BG composition (called Bioglass $^{\circledR}$ or 45 S5) lacks fluoride ${ }^{9}$, so some researchers have added fluoride to it and demonstrated superior remineralization competence to other toothpastes ${ }^{10)}$.

Color figures can be viewed in the online issue, which is available at J-STAGE.

Received Mar 11, 2020: Accepted May 13, 2020

doi:10.4012/dmj.2020-078 JOI JST.JSTAGE/dmj/2020-078
Theobromine, an alkaloid, is readily available in chocolate products and cocoa ${ }^{11)}$. It is reported that its presence in an apatite-forming medium could enhance the medium's potential to reverse demineralization ${ }^{12)}$. Theobromine-containing toothpastes like Theodent ${ }^{\mathbb{B}}$ (New Orleans, LA, USA) are available in the market; however, they do not contain any fluoride ${ }^{12)}$.

The authors could not find any study where theobromine has been incorporated with $\mathrm{BG}$ in a toothpaste. The combination of theobromine with a fluoridated BG (F-BG) to develop a toothpaste would be interesting, as all three of these ingredients have proven remineralizing capabilities. Therefore, the aim of this study was to develop a novel toothpaste containing theobromine with F-BG and to investigate its effect on physical and mechanical properties of human enamel. The null hypothesis $\left(\mathrm{H}_{0}\right)$ was set to be that all the toothpastes in this study (commercial and experimental) would result in similar increase in enamel's microhardness and decrease in its surface roughness posttreatment.

\section{MATERIALS AND METHODS}

Ethical approval (Ref: EA 2019041) was obtained from the scientific research unit of the college, and all ethical protocols were strictly followed. The study was conducted in two stages, where the first stage involved preparation of a basic paste and then addition of various active ingredients to it to attain a novel toothpaste. The second stage involved its testing against various commercial brands of toothpaste on extracted teeth to determine its effect on physical and mechanical properties of enamel. The group containing basic paste only was used 
as a positive control group, whereas Theodent ${ }^{\circledR}$ and Sensodyne $^{\circledR}$ (GSK, UK) were used as negative control groups for theobromine and $\mathrm{BG}$, respectively.

\section{Preparation of basic paste}

All the chemicals and precursors were of analytical grade and were commercially purchased from SigmaAldrich, USA. In this study, initially, a basic paste was prepared by using basic ingredients. In $100 \mathrm{~mL}$ of distilled water, $1.3 \mathrm{~g}$ of calcium carbonate was mixed inside a magnetic stirrer at room temperature. The mixture was left in stirring mode for $2 \mathrm{~h}$. Glycerol (16 $\mathrm{mL}$ ) was then added to the mixture, which was stirred for $1 \mathrm{~h}$. After complete mixing, $0.5 \mathrm{~g}$ of sodium benzoate was added, and the mixture was stirred for $40 \mathrm{~min}$. Then, $0.5 \mathrm{~g}$ of methylcellulose was added, and the mixture was again stirred for $40 \mathrm{~min}$. After the addition of all basic ingredients, a flavouring agent $(0.5 \mathrm{~mL}$ of peppermint oil) was added, and the components were mixed overnight for complete mixing of powders in the paste.

\section{Preparation of active ingredients}

Two active ingredients were added to the basic paste: theobromine and F-BG nanoparticles. Theobromine (AKSci $\left.{ }^{\circledR}, \mathrm{CA}, \mathrm{USA}\right)$ was commercially acquired, whereas F-BG was synthesized by using calcium nitrate $\left(\mathrm{Ca}\left(\mathrm{NO}_{3}\right)_{2} \cdot 4 \mathrm{H}_{2} \mathrm{O}\right)$ and diammonium hydrogen phosphate $\left(\left(\mathrm{NH}_{4}\right)_{2} \mathrm{HPO}_{4}\right)$ as sources of calcium and phosphate precursors, respectively. Initially, 11.7 mmol of $\mathrm{Ca}\left(\mathrm{NO}_{3}\right)_{2} \cdot 4 \mathrm{H}_{2} \mathrm{O}$ and $23.1 \mathrm{mmol}$ of triethyl orthosilicate (TEOS) were dispersed in $25 \mathrm{~mL}$ ethanol solution. During this reaction, the $\mathrm{pH}$ was maintained at $1-2$ by adding nitric acid. Then, $2.47 \mathrm{mmol}$ of sodium fluoride $(\mathrm{NaF})$ was added to the reaction and allowed to mix. Later, this solution was added slowly to 2.86 mmol diammonium hydrogen phosphate $\left(\left(\mathrm{NH}_{4}\right)_{2} \mathrm{HPO}_{4}\right)$ solution. The $\mathrm{pH}$ of solution was kept at 11 during the reaction using ammonia. After stirring for $48 \mathrm{~h}$, the solution was filtered, washed to neutralize the $\mathrm{pH}$, and dried at $70^{\circ} \mathrm{C}$. The obtained sample was heat treated at $500^{\circ} \mathrm{C}$ for $6 \mathrm{~h}$ at the heating rate of $2^{\circ} \mathrm{C} \cdot \mathrm{min}^{-1}$.

Addition of active ingredients to basic paste Theobromine (0.2 wt\%) and freshly synthesized F-BG (4 wt\%) were added separately to the basic paste in increments, and the mixture was stirred overnight in a magnetic stirrer at room temperature. For the experimental toothpaste, $0.2 \mathrm{wt} \%$ theobromine was added to the basic paste and allowed to stir for $24 \mathrm{~h}$ to ensure uniform mixing. Then, F-BG (4 wt\%) was added in increments and stirred overnight for another $24 \mathrm{~h}$.

\section{Preparation of enamel blocks}

Thirty-six freshly extracted teeth were collected from the Oral Surgery department of the institute. Teeth which were free from white spot lesions, caries, restorations, and other evident defects were selected for this study. The anatomical crowns of the teeth were obtained by cutting the teeth over the cemento-enamel junction utilizing a water-cooled diamond saw (Isomet ${ }^{\mathbb{P}}$ 5000 Linear Precision Saw, Buehler, IL, USA). After discarding the roots, the crowns were embedded in selfcure acrylic resin inside a pre-fabricated square mold to facilitate standardization. The crowns were placed in such a manner that their buccal/labial enamel surfaces were exposed, whereas the lingual surface was in contact with the self-cure resin. On the exposed enamel surface, a window of approximately $4 \times 4 \mathrm{~mm}$ was created with a nail varnish to ensure that all brushing experiments and analysis were done only in that particular area. The exposed enamel surfaces were polished with 600grit wet silicon-carbide paper for $1 \mathrm{~min}$. Then, the enamel samples were subjected to citric acid challenge to induce demineralization. The enamel samples were immersed in $6 \mathrm{wt} \%$ citric acid $(\mathrm{pH}=2.0)$ for 5 min inside a glass beaker, which was placed on top of a rotating orbital shaker (CO-Z ${ }^{\circledR}$ Orbital Shaker, USA) to mimic invivo conditions. After the acidic challenge, the enamel samples were washed with distilled water for $1 \mathrm{~min}$ and then left to air-dry.

These enamel blocks were randomly divided into six groups, with each group receiving six specimens that received brushing with $1.0 \mathrm{~g}$ of their respective toothpaste mixed with AS (in a 1:2 ratio) as shown in Table 1.

\section{Artificial saliva (AS) preparation}

The AS was prepared by mixing $0.4 \mathrm{~g} \mathrm{NaCl}, 0.4 \mathrm{~g} \mathrm{KCl}$, $0.6 \mathrm{~g} \mathrm{NaH}_{2} \mathrm{PO}_{4} \cdot \mathrm{H}_{2} \mathrm{O}, 0.79 \mathrm{~g} \mathrm{CaCl} \cdot \mathrm{H}_{2} \mathrm{O}$, and $0.005 \mathrm{~g}$ $\mathrm{Na}_{2} \mathrm{~S} \cdot 9 \mathrm{H}_{2} \mathrm{O}$ in $1,000 \mathrm{~mL}$ of deionized water, as suggested earlier by Fusayama et $a l .{ }^{13)}$. The $\mathrm{pH}$ of AS was 5.5 , which was adjusted to a neutral $\mathrm{pH}$ of 7 by utilizing $\mathrm{NaOH}$.

Table1 Showing brushing treatment of enamel blocks by their respective toothpastes for various groups in this study

\begin{tabular}{cl}
\hline Groups & \multicolumn{1}{c}{ Brushing treatment of enamel blocks by their respective toothpastes } \\
\hline Group 1 & Enamel blocks brushed with basic paste \\
Group 2 & Enamel blocks brushed with basic paste $+0.2 \mathrm{wt} \%$ theobromine toothpaste \\
Group 3 & Enamel blocks brushed with commercial theobromine toothpaste \\
Group 4 & Enamel blocks brushed with commercial BG toothpaste \\
Group 5 & Enamel blocks brushed with basic paste $+4 \mathrm{wt} \% \mathrm{~F}-\mathrm{BG}$ toothpaste \\
Group 6 & Enamel blocks brushed with basic paste $+0.2 \mathrm{wt} \%$ theobromine +4 wt\% F-BG toothpaste \\
\hline
\end{tabular}




\section{Tooth brushing protocol}

Manual toothbrushes belonging to one commercial brand (Trisa ${ }^{\circledR}$, Switzerland) were used. A toothbrushing simulator (Toothbrush Simulator; model ZM-3.8, Germany) was used to replicate toothbrushing, giving each sample 5,000 linear strokes, which is equivalent to 6 months of in vivo brushing ${ }^{1)}$. The applied load was 250 $\mathrm{g}$, and the linear distance covered by toothbrushes was set to $50 \mathrm{~mm}$. After every 500 strokes, $1.0 \mathrm{~g}$ of respective toothpaste mixed with $\mathrm{AS}$ in a 1:2 ratio was added to the sample. After the brushing strokes, the samples were washed with distilled water for $1 \mathrm{~min}$ and were left to air-dry before further analysis.

\section{Surface micro-hardness analysis}

The Vickers hardness data of the enamel surface were collected at three time intervals: pre-treatment, postdemineralization, and post-treatment. The specimens from each group were evaluated for changes in surface micro-hardness values. The Vickers hardness was measured using a digital micro-hardness tester (FM-ARS 9000; Future-Tech, Kawasaki, Japan). Three indents were made on the treated surface of each specimen using a Vickers diamond indenter under a $100 \mathrm{~g}$ load applied for $10 \mathrm{~s}$.

\section{Surface roughness measurement}

Surface roughness was measured three times with a Contour GT Optical Microscopes profilometer (Bruker, Tucson, AZ, USA) at three intervals (pre-treatment, post-demineralization, and post-treatment), just like in the surface micro-hardness analysis. Each specimen was placed in a marked fixed position so that the scan was performed in the exposed enamel window area only. The mean surface roughness values $(\mathrm{Ra})$ (which are the mean of the height of peaks and the depth of valleys from the mean line along the measuring length) were then calculated for all the samples.

\section{Statistical analysis}

The results were analyzed using Statistical Package for the Social Sciences (SPSS) software (version 19.0; SPSS, Chicago, IL, USA). The Wilcoxon sign-rank test was applied to evaluate significant differences within each group comparing pre-treatment vs post-demineralization values and pre-treatment vs post-treatment values. A $p$-value $\leq 0.05$ was considered statistically significant.

\section{RESULTS}

\section{Surface micro-hardness analysis}

The Vickers hardness number (VHN) was obtained by subtracting the post-treatment values from postdemineralization values. The results indicate that group 6 performed best, followed by group 4, group 2 , and group 5 (Table 2). Groups 1 and 3 showed a further decrease in micro-hardness of enamel samples (Table 2). All intra-group comparisons were statistically significant when pre-treatment $v s$ post-demineralization values were paralleled, whereas post-demineralization $v s$ posttreatment values were significant for groups $1-3$ and 6 .

\section{Surface roughness analysis}

The enamel surface profile was revealed by profilometry for groups 1-3, as shown in Fig. 1, whereas for groups 4-6, it is shown in Fig. 2. These images demonstrate enamel surface profile post-polishing (a), post-demineralization (b), and post-treatment (c). The surface roughness results (values in $\mu \mathrm{m}$ ) show the maximum decrease in roughness values for group 6 , followed by group 5 and group 1 (Table 3). For all the other groups, roughness values were increased post-brushing, as tabulated in Table 3. Intra-group comparisons were significant for groups $2-6$ when pre-treatment $v$ s post-demineralization values were compared, whereas they were significant for groups $2-5$ when post-demineralization values were matched against post-treatment values.

Table 2 Showing mean VHN values (SD) for all groups pre-treatment, post-demineralization, post-treatment

\begin{tabular}{|c|c|c|c|c|c|c|}
\hline & \multicolumn{6}{|c|}{ Group statistics mean VHN (SD) } \\
\hline & Group-1 & Group-2 & Group-3 & Group-4 & Group-5 & Group-6 \\
\hline Pre-treatment & $520(83)$ & $558(52)$ & $546(49)$ & $577(123)$ & $540(75)$ & $554(29)$ \\
\hline Post-demineralization & $406(73)$ & $330(38)$ & $398(75)$ & $480(131)$ & $473(35)$ & $399(34)$ \\
\hline Post-treatment & $404(65)$ & $357(53)$ & $359(62)$ & $522(90)$ & $492(42)$ & $482(52)$ \\
\hline${ }^{\wedge} p$-value & 0.028 & 0.028 & 0.028 & 0.028 & 0.028 & 0.028 \\
\hline${ }^{\wedge} p$-value & 0.046 & 0.028 & 0.028 & 0.345 & 0.600 & 0.028 \\
\hline $\begin{array}{l}\text { Difference } \\
\text { (post-treatment-post } \\
\text { demineralization) }\end{array}$ & -2 & 27 & -39 & 42 & 19 & 83 \\
\hline
\end{tabular}

^p-value: Within group significance pre-treatment versus post-demineralization

${ }^{\wedge} p$-value: Within group significance pre-treatment versus post-treatment

A "-ve" value demonstrates reduction in micro-hardness 

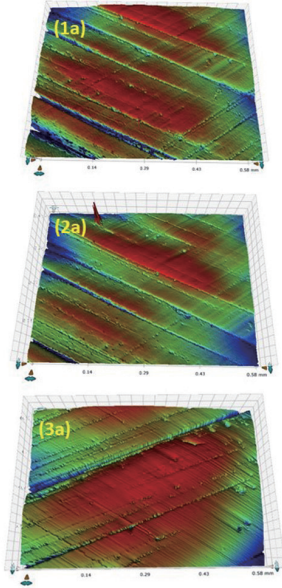
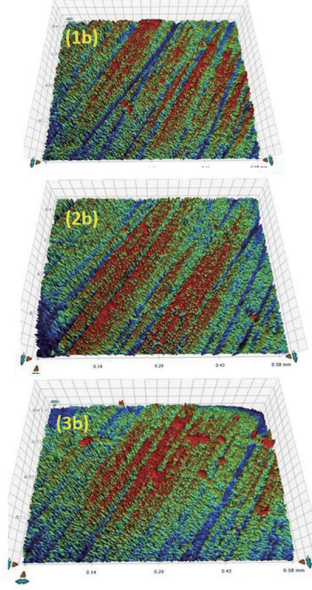
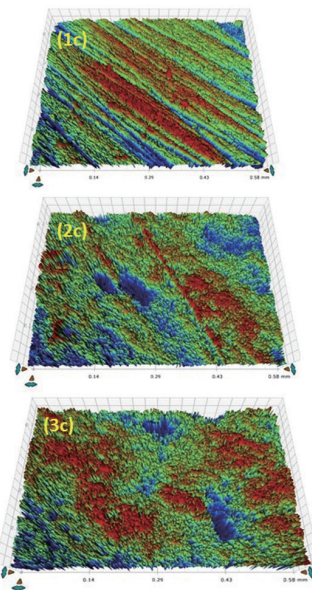

Fig. 1 Three dimensional (3D) profilometric images belonging to groups 1-3.

a) Pre-treatment, b) Post-demineralization, and c) Post-treatment and showing Ra values
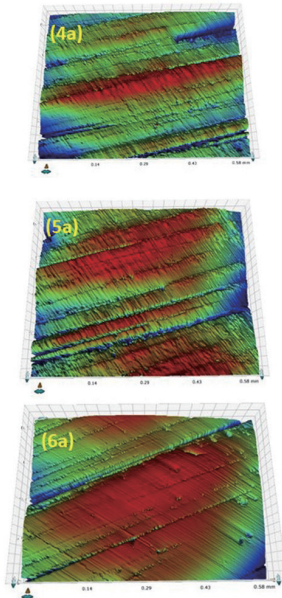
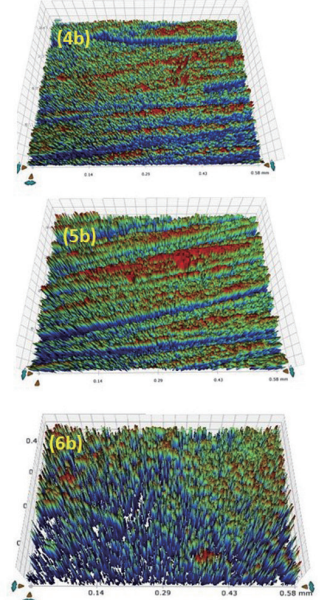
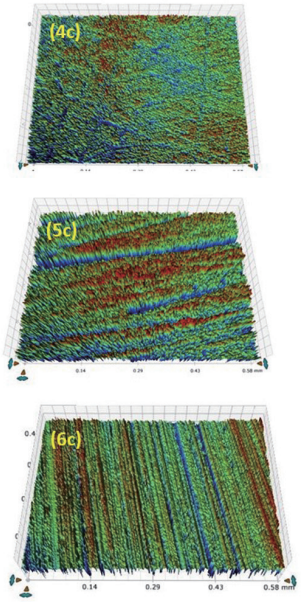

Fig. 2 3D profilometric images belonging to groups 4-6.

a) Pre-treatment, b) Post-demineralization, and c) Post-treatment and showing Ra values

Table 3 Showing mean Ra values for all groups pre-treatment, post-demineralization, post-treatment

\begin{tabular}{lcccccc}
\hline & \multicolumn{7}{c}{ Group statistics mean Ra $(\mu \mathrm{m})(\mathrm{SD})$} \\
\cline { 2 - 7 } & Group-1 & Group-2 & Group-3 & Group-4 & Group-5 & Group-6 \\
\hline Pre-treatment & $0.86(0.86)$ & $0.44(0.20)$ & $0.52(0.25)$ & $0.39(0.08)$ & $0.59(0.18)$ & $0.62(0.17)$ \\
Post-demineralization & $0.93(0.41)$ & $1.09(0.33)$ & $1.51(0.92)$ & $1.54(0.91)$ & $1.65(1)$ & $1.96(1.66)$ \\
Post-treatment & $0.74(0.29)$ & $1.15(0.63)$ & $1.63(0.78)$ & $2.11(2.65)$ & $1.12(0.42)$ & $0.55(0.08)$ \\
${ }^{\wedge} p$-value & 0.345 & 0.028 & 0.028 & 0.028 & 0.028 & 0.028 \\
^ $p$-value & 0.345 & 0.028 & 0.028 & 0.028 & 0.046 & 0.463 \\
$\begin{array}{l}\text { Difference } \\
\text { (post treatment-post } \\
\text { demineralization) }\end{array}$ & -0.19 & 0.06 & 0.12 & 0.53 & -0.53 & -1.41 \\
\hline
\end{tabular}

${ }^{\wedge} p$-value: Within group significance pre-treatment versus post-demineralization.

${ }^{\wedge} p$-value: Within group significance pre-treatment versus post-treatment.

A "-ve" value demonstrates reduction in surface roughness. 


\section{DISCUSSION}

Based on the results of this study, $\mathrm{H}_{0}$ was rejected, as the results indicate superior performance of the experimental toothpaste with theobromine and F-BG as compared with others in terms of increasing micro-hardness and decreasing surface roughness of demineralized human enamel.

The purpose of this study was to prepare a toothpaste containing theobromine with F-BG and then evaluate its competence to increase microhardness and decrease surface roughness following demineralization. In this study, 0.2 wt $\%$ and 4 wt\% of theobromine and F-BG were used in the experimental toothpaste respecitvely. These percentages were obtained from previously conducted studies that showed positive outcome of these materials on enamel and dentin tissues, without any toxic effect ${ }^{1,14)}$. Clinically, the appearance of early enamel carious lesion is white, which occurs as a result of loss of normal translucency of the enamel ${ }^{15)}$. As a consequence of caries initiation, the enamel surface has lower mineral content in comparison to a sound enamel surface; therefore, the affected layer possesses a lower hardness value when compared with a sound enamel surface $^{16)}$. Hence, clinically, the surface of the enamel becomes fragile as a result of carious lesion and is quite vulnerable to breakage on probing.

Considering the significance of the surface enamel layer in relation to caries development, the assessment of this layer is of utmost importance. Micro-hardness measurement is a tool that provides a simple, fast, and non-invasive way to evaluate the hardness of a surface that is prone to cracks and fractures, such as enamel $^{17)}$. The micro-hardness values analyzed by calculating VHN recorded that pre-treatment values in this study were in the range of 520-577, which was slightly higher than the microhardness value reported by other studies ${ }^{18,19)}$. This increased pre-treatment VHN value could be a result of teeth being used which might have been exposed to fluoridated toothpastes prior to extraction, as opposed to freshly erupted teeth. It should also be noted that high standard deviation values were observed on micro-hardness analysis. The probable reason for this could be non-homogeneity of material surface (enamel), as the teeth were collected from different patients in oral surgery department. Following the acidic challenge, the micro-hardness values of the enamel specimens from each group decreased, and this is in agreement with previously reported studies ${ }^{20,21)}$. The present study showed that the highest micro-hardness value was achieved by the experimental toothpaste containing theobromine with F-BG, although samples went through a demineralization process first, which is a fairly vigorous challenge. This could be the result of the presence of three remineralizing agents together in this experimental toothpaste, namely theobromine, fluoride, and BG. Theobromine can be inserted in the microtunnel that is found within hydroxyapatite crystals of enamel, thus causing an internal stress that requires a greater external stress to cause deformation ${ }^{22}$. This in turn increases the strength and hardness of the enamel surface. Fluoride and BG have proven their ability to remineralize the enamel structure in various previously carried out studies ${ }^{16,20)}$. Although the presence of these agents alone in other groups caused an increase in micro-hardness, it was still less than in the experimental toothpaste group, accentuating that the presence of these agents together could lead to an improved and harder enamel surface that is resistant to acidic challenges.

Apart from surface micro-hardness, non-contact profilometry or surface roughness was also used in this study, as researchers believe that it is a good tool to assess dental hard tissue's surface loss or gain. In the present study, the maximum decrease in roughness was observed with the experimental toothpaste containing theobromine and F-BG. There is a scarcity of studies in the literature that have evaluated the effect of theobromine in reducing enamel's surface roughness, and to the best of the authors' knowledge, this is the first study to evaluate this. In the current study, neither a commercially available toothpaste containing theobromine nor its combination with basic paste reduced the roughness of the demineralized enamel surface. However, its combination with F-BG decreased surface roughness significantly, even more than the combination of BG with basic paste. The mechanism by which BG reduces surface roughness is well understood and exceedingly reported in the scientific literature ${ }^{23,24)}$, however, theobromine's exact mechanism of reducing enamel's roughness has not been reported. The only information available regarding it is in a previous study that stated that addition of theobromine could increase the enamel crystal size, subsequently inhibiting surface dissolution of ions ${ }^{14)}$, thus making the surface less rough, as was observed in the present study.

This study had a few limitations, the first of which was its in-vitro nature. Although every attempt was made to ensure standardization of experiments, the results of this study should still be interpreted cautiously, as in-vivo conditions are dynamic and could be different from an in-vitro setting. Another limitation was that the hardness level and roughness values could be diverse in different enamel surfaces; still, an attempt was made to perform pre-treatment, post-demineralization, and post-treatment scans in the designated marked area on the enamel's surface. Future quantitative and clinical studies should be performed to test the efficacy of our experimental toothpaste under more vibrant in-vivo conditions.

\section{CONCLUSION}

Within the limitations of this study, it is concluded that toothpaste containing $0.2 \mathrm{wt} \%$ theobromine $+4 \mathrm{wt} \% \mathrm{~F}-\mathrm{BG}$ exhibited better results than the other groups in terms of increasing micro-hardness of enamel and decreasing its roughness. Therefore, toothpaste containing $0.2 \mathrm{wt} \%$ theobromine +4 wt $\%$ F-BG can be suggested as regular use toothpaste, especially for patients at high risk of caries and erosion. 


\section{ACKNOWLEDGMENTS}

The authors are grateful to Deanship of Scientific Research, Imam Abdulrahman Bin Faisal University, Dammam, Saudi Arabia for providing funding for this study (Grant no. 2017-395-Dent). The authors are also thankful to Mr. Intisar Ahmed Siddiqui for helping us with the statistical analysis.

\section{CONFLICT OF INTEREST}

The authors declare that there is no conflict of interest.

\section{REFERENCES}

1) Khan AS, Farooq I, Alakrawi KM, Khalid H, Waqas O, Hakeem AS. Dentin tubule occlusion potential of novel dentifrices having fluoride containing bioactive glass and zinc oxide nanoparticles. Med Princ Pract 2020; 29: 338-346.

2) Featherstone JD. Dental caries: a dynamic disease process. Aus Dent J 2008; 53: 286-291.

3) Khoroushi M, Kachuie M. Prevention and treatment of white spot lesions in orthodontic patients. Contemp Clin Dent 2017; 8: 11-19.

4) Farooq I, Moheet IA, Alshwaimi E. In vitro dentin tubule occlusion and remineralization competence of various toothpastes. Arch Oral Biol 2015; 60: 1246-1253.

5) Clark MB, Slayton RL, Hlth SO. Fluoride use in caries prevention in the primary care setting. Pediatrics 2014; 134 : 626-633.

6) Aoba T. The effect of fluoride on apatite structure and growth. Crit Rev Oral Biol Med 1997; 8: 136-153.

7) Mohammed NR, Lynch RJ, Anderson P. Effects of fluoride concentration on enamel demineralization kinetics in vitro. $\mathrm{J}$ Dent 2014; 42: 613-618.

8) Jones JR. Review of bioactive glass: from Hench to hybrids. Acta Biomater 2013; 9: 4457-4486.

9) Ali S, Farooq I, Iqbal K. A review of the effect of various ions on the properties and the clinical applications of novel bioactive glasses in medicine and dentistry. The Saudi Dent $J$ 2014; 26: 1-5.

10) Bakry AS, Abbassy MA, Alharkan HF, Basuhail S, Al-Ghamdi K, Hill R. A novel fluoride containing bioactive glass paste is capable of re-mineralizing early caries lesions. Materials 2018; 11: 1636.

11) Amaechi BT, Mathews SM, Mensinkai PK. Effect of theobromine-containing toothpaste on dentin tubule occlusion in situ. Clin Oral Invest 2015; 19: 109-116.

12) Amaechi BT, Porteous N, Ramalingam K, Mensinkai PK, Vasquez RAC, Sadeghpour A, et al. Remineralization of artificial enamel lesions by theobromine. Caries Res 2013; 47: 399-405.

13) Fusayama T, Nomoto S, Katayori T. Corrosion of gold and amalgam placed in contact with each other. J Dent Res 1963; 42: 1183-1197.

14) Kargul B, Ozcan M, Peker S, Nakamoto T, Simmons WB, Falster AU. Evaluation of human enamel surfaces treated with theobromine: a pilot study. Oral Health Prev Dent 2012; 10: $275-282$.

15) Zhang J,Lynch RJM, Watson TF, BanerjeeA. Remineralisation of enamel white spot lesions pre-treated with chitosan in the presence of salivary pellicle. J Dent 2018; 72: 21-28.

16) Lata S, Varghese NO, Varughese JM. Remineralization potential of fluoride and amorphous calcium phosphate-casein phospho peptide on enamel lesions: An in vitro comparative evaluation. J Conserv Dent 2010; 13: 42-46.

17) Zhang YR, Du W, Zhou XD, Yu HY. Review of research on the mechanical properties of the human tooth. Int J Oral Sci 2014; 6: 61-69.

18) Craig RG, Peyton FA. The micro-hardness of enamel and dentin. J Dent Res 1958; 37: 661-668.

19) Palti DG, Machado MA, Silva SM, Abdo RC, Lima JE. Evaluation of superficial microhardness in dental enamel with different eruptive ages. Braz Oral Res 2008; 22: 311315.

20) Alhussain AM, Alhaddad AA, Ghazwi MM, Farooq I. Remineralization of artificial carious lesions using a novel fluoride incorporated bioactive glass dentifrice. Dent Med Prob 2018; 55: 379-382.

21) Mudumba VL, Muppa R, Srinivas N, Kumar DM. Evaluation and comparison of changes in microhardness of primary and permanent enamel on exposure to acidic center-filled chewing gum: an in vitro study. Int J Clin Ped Dent 2014; 7: 24-29

22) Pribadi N, Citra A, Rukmo M. The difference in enamel surface hardness after immersion process with cocoa rind extract (Theobroma cacao) and fluoride. J Int Oral Health 2019; 11: 100-103.

23) Taha AA, Fleming PS, Hill RG, Patel MP. Enamel remineralization with novel bioactive glass air abrasion. J Dent Res 2018; 97: 1438-1444.

24) Dionysopoulos D, Tolidis K, Sfeikos T. Effect of air-abrasion pre-treatment with bioactive glass $45 \mathrm{~S} 5$ on enamel surface loss after erosion/abrasion challenge. Dent Mater 2019; 35: e193-e203. 